\title{
IL-17 Activates the IL-6/STAT3 Signal Pathway in the Proliferation of Hepatitis B Virus-Related Hepatocellular Carcinoma
}

\author{
Zongqiang Hu Ding Luo Dongdong Wang Linjie Ma Yingpeng Zhao Li Li \\ The First People's Hospital of Kunming City \& Ganmei Affiliated Hospital of Kunming Medical \\ University, Kunming, China
}

\section{Key Words}

$\mathrm{IL}-17 \cdot \mathrm{IL}-6 \cdot \mathrm{pSTAT} 3 \cdot \mathrm{HBV} \cdot \mathrm{HCC}$

\begin{abstract}
Background/Aims: We performed this study to determine the role of IL-17 in the immune microenvironment of hepatitis B virus- (HBV-) related hepatocellular carcinoma (HCC). Methods: HepG2 cells were treated with IL-17, STAT3 inhibitor S31-201 or IL-6 neutralizing monoclonal antibody (IL-6 mAb). Cell proliferation and migration were compared using the Cell Counting kit-8 (CCK-8) and Transwell assays, respectively. Real-time quantitative PCR (RT-qPCR), Western Blot, ELISA, immunofluorescence and histological staining were used for determining the expression levels of IL-17, IL-6, MCP-1, CCL5, VEGF, STAT3 and p-STAT3. HCC xenograft models were constructed in wild type and IL-17 knockout mice to clarify the effects of IL-17 on HCC in vivo. Results: Exogenous IL-17 enhanced the proliferation and migration of HepG2 cells, and it activated the phosphorylation of STAT3. RT-qPCR and ELISA showed that IL-17 promoted the expression of IL- 6 . The CCK-8 and Transwell assays showed that S31201 or IL-6 mAb remarkably reversed the promotion effects of proliferation and migration by exogenous IL-17 in HepG2 cells. Additionally, IL-6 could promote the phosphorylation of STAT3, while IL- 6 mAb acted as an inhibitor, and exogenous IL-17 could neutralize the inhibitory effects of IL-6 mAb. In vivo, compared to the wild type mice, the tumor volume, weight, density and size were decreased in IL-17 knockout mice. Additionally, the expression levels of p-STAT3, IL-6, MCP-1, CCL5 and VEGF decreased in IL-17 knockout mice. Conclusions: IL-17 can enhance the proliferation of HepG2 cells in vitro and in vivo via activating the IL-6/ STAT3 pathway. Therefore, the IL-17/IL-6/STAT3 signaling pathway is a potential therapeutic target for HBV-related HCC.

\section{Introduction}

Hepatocellular carcinoma (HCC), the third most prevalent cause of cancer-related death across the world, is one of the five most common cancers [1]. Approximately 20,000 estimated new HCC cases in the United States were reported in 2010 [2]. A number of studies showed that hepatitis B virus (HBV) infection, excess alcohol consumption, exposure 
to aflatoxin and smoking are the major risk factors of HCC [3], and persistent HBV infection is the most dangerous and prevalent cause [4]. Hepatic carcinogenesis, triggered by HBV, involves complicated mechanisms, which are related to immune responses induced by HBV infection and replication, such as inflammatory cytokine expression [5].

Variation in the clinical outcomes of HBV infection and the disease course of HCC may result from numerous immuno-inflammatory cells, cytokines and chemokines [6]. Among the inflammatory signaling pathways that are related to tumor development, the phosphorylation of signal transducer and activator of transcription (STAT3) plays a key role [7]. STAT3, an oncogenic transcription factor, is usually constitutively active in many human cancers, such as prostate cancer, breast cancer and several other malignancies [8-10]. STAT3 has been considered a target for anti-cancer therapy because it plays an important role in tumor cell survival and proliferation [11]. The STAT3 molecule is also responsible for anti-apoptosis activity and cell proliferation of HCC cells [12]. Additionally, STAT3 can be activated by interleukin-6 (IL-6) [13]. Indeed, the activation of IL-6/STAT3 is considered a "bona fide" tumor promoter in obesity [14] and fatty liver-associated inflammation [15].

Interleukin-17 (IL-17) is an essential pro-inflammatory cytokine, which consists of six family ligands, [16]. Previous studies have reported that IL-17 plays an important role in various chronic diseases, including $[17,18]$ inflammatory bowel disease, rheumatoid arthritis, and multiple sclerosis [19]. Recently, evidence has indicated that IL-17 is related to many human cancers, including gastric cancer, breast cancer, and ovarian cancer [20-22]. IL17 may contribute to HBV-associated liver diseases, and it can stimulate HCC cell migration and invasion [23]. Additionally, Gu et al. found that IL-17 expression was significantly associated with the phosphorylation of STAT3 [24]. Although the correlation between IL17 and the risk of postoperative recurrence and poor survival of HCC have been verified in previous studies, the underlying mechanisms of IL-17 in modulating HCC cell growth remain elusive [24].

In this study, IL-17 deficient mouse models are used and various experiments are performed to investigate the role of IL-17 in hepatic carcinogenesis induced by HBV. In addition, the interaction between IL-17 and the IL-6/STAT3 signaling pathway is discussed. Through these experiments, our research objective is to identify an alternative target for treating HCC.

\section{Materials and Methods}

\section{Cell lines}

The cell lines used in the experiment were HepG2 $\left(\mathrm{HBV}^{+} \mathrm{HBsAg}^{+}\right.$, human hepatocellular carcinoma cells, purchased from the Institute of Biochemistry and Cell Biology in Shanghai), which were cultured in Dulbecco's modified eagle medium (DMEM). The DMEM in our experiment contained $100 \mu \mathrm{g} / \mathrm{mL}$ streptomycin (HyClone, South Logan, UT, USA), 10\% fetal bovine serum (FBS), $100 \mathrm{U} / \mathrm{mL}$ penicillin, and 250 $\mu \mathrm{g} / \mathrm{mL}$ G418 (Sigma, St. Louis, MO, USA). All cells were incubated at $37^{\circ} \mathrm{C}$ in $5 \% \mathrm{CO}_{2}$. The culture solution was replaced by new media every 2 days. Recombinant human IL-17 (R\&D Systems, Minneapolis, MN, USA), an IL-6 neutralizing monoclonal antibody (IL-6 mAb, R\&D Systems) and STAT3 inhibitor S31-201 (Selleck, Houston, TX, USA) were applied as indicated.

Cell Counting kit-8 (CCK-8) Test

Cells were seeded onto 96-well plates $\left(2 \times 10^{3}\right.$ cells per well) and cultured for 1 to 3 days in medium supplemented with recombinant human IL-17 (0, 5, 50100,250 and $500 \mathrm{ng} / \mathrm{mL})$. After IL-17 treatment, 10 $\mu \mathrm{L}$ of CCK-8 (Zomanbio, Beijing, China) was added to each well, which was followed by incubation for $3 \mathrm{~h}$. The absorbance at $450 \mathrm{~nm}$ for each well was read on a spectrophotometer.

Transwell Assay

The HepG2 cells $\left(3 \times 10^{5} / \mathrm{mL}\right)$ treated with recombinant human IL-17, STAT3 inhibitor S31-201 or IL-6 mAb were cultured for $24 \mathrm{~h}$ were suspended in DMEM without serum and then added into the upper KARGER 


\section{Cellular Physiology Cell Physiol Biochem 2017;43:2379-2390 \begin{tabular}{l|l|l} 
and Biochemistry 10.1159/000484390 & $\begin{array}{l}\text { (c) 2017 The Author(s). Published by S. Karger AG, Basel } \\
\text { www.karger.com/cpb }\end{array}$
\end{tabular}}

Hu et al.: IL-17/IL-6/STAT3 in HBV-Related HCC

chamber of a 6.5-mm Transwell, and the lower chamber was filled with $600 \mu \mathrm{L}$ DMEM containing $10 \%$ FBS. After incubation for $24 \mathrm{~h}$ at $37^{\circ} \mathrm{C}$, the transmembrane cells were stained with $0.1 \%$ crystal violet. Five point fields were randomly selected to count the numbers of stained cells by microscopy (Olympus, Tokyo, Japan).

\section{Western Blot}

HepG2 cells $\left(1 \times 10^{5}\right)$ treated with recombinant human IL-17, STAT3 inhibitor S31-201 or IL-6 mAb were cultured for $24 \mathrm{~h}$. Total protein was extracted from the cells and samples, and the protein concentration was determined by the bicinchoninic acid (BCA) method (Thermo Fisher Scientific, Waltham, MA, USA). Equal levels of protein were separated with sodium dodecyl sulfate polyacrylamide gel electrophoresis (SDS-PAGE) gels and subsequently transferred to nitrocellulose membranes; then, the membranes were blocked with $5 \%$ nonfat milk for $2 \mathrm{~h}$ at room temperature and incubated overnight at $4{ }^{\circ} \mathrm{C}$ with primary antibodies for STAT3 (ab68153, Abcam, UK, 1:900), p-STAT3 (E121-31, Abcam, 1:1200) and $\beta$-actin (ab8227, Abcam, 1:700); $\beta$-actin was used for normalization. Then, incubation with secondary antibodies (Beyotime Biotechnology, Shanghai, China) for another $2 \mathrm{~h}$ was followed by washing with phosphate buffer (PBS). The signals were detected using the enhanced chemiluminescence substrate kit (Thermo Fisher Scientific). Protein bands were semi-quantified by ImageJ software.

\section{$R T-q P C R$}

Total RNA extraction and reverse transcription were performed as previously described [25]. For frozen tissues, Trizol reagent (Beyotime Biotechnology, China) was used to dissolve the tissues after they were processed. Total RNA was reverse transcribed into cDNA according to the manufacturer's protocol for the RT Kit (\#K1622, Fermentas, MA, USA). RT-qPCR was performed using a PCR kit (C10572-014, Invitrogen, Carlsbad, CA, USA) and a CFX96 Touch Real-Time PCR Detection System (Bio-Rad, Hercules, CA, USA). All primers were synthesized by Shanghai GenePharma Co., Ltd., and the sequences are shown in Table 1. GAPDH was used as an internal control to normalize the IL-6 expression. Differential expression of IL-6 was calculated using the $2^{-\triangle \Delta C T}$ method.

\section{Enzyme linked immunosorbent assay (ELISA) analysis}

HepG2 cells $\left(1 \times 10^{5}\right)$ transfected with recombinant human IL-17 were incubated and cultured for 24 $\mathrm{h}$; then, culture supernatants were collected. The protease inhibitor was added into the liver tumor and then tissues were homogenized. After $30 \mathrm{~min}$ of $12000 \mathrm{r} / \mathrm{min}$ centrifugation, total protein was extracted from the supernatant. The levels of IL-6, MCP-1, CCL5 and VEGF were determined using ELISA kits (R\&D Systems) according to the manufacturer's instructions and then quantified by a microplate reader ( $450 \mathrm{~nm})$.

\section{Immunofluorescence Microscopy}

HepG2 cells were collected by trypsin and seeded in glass slides (Thermo Scientific) at a density of 2-5 $\times 10^{3}$. After cultivation for $48 \mathrm{~h}$, cells were fixed with $4 \%$ paraformaldehyde for $15 \mathrm{~min}$ and then incubated with primary antibodies for STAT3 (Abcam, 1:50) and p-STAT3 (Abcam, 1:50) overnight at $4{ }^{\circ} \mathrm{C}$, which was followed by incubation with secondary Alexa fluor 488-labeled (green) anti-IgG or Alexa fluor 555 labeled (red) anti-IgG (Invitrogen). DAPI was used to stain the nuclei. Confocal fluorescence microscopy was used to observe and photograph fluorescent sections.

\section{Heterotopic xenograft liver tumor models}

We selected healthy and specific pathogen-free BALB/C mice (5-week-old, female, average body weight of $25 \mathrm{~g}$, purchased from Shanghai Silaike Experimental Animal Co., Ltd.) for the control group. IL-17 KO mice (5-week-old, female, average weight of $26 \mathrm{~g}$ ) were purchased from Tokyo University and were fed in the Shanghai Southern Research Center. All mice in the group were water and food ad libitum, and they were housed in 12-h light/dark cycles until the date of the experiment. All experiments were performed in accordance with the Institutional Animal Care and Use Committee (IACUC) management protocol.

Table 1. Primer sequences of GADPH and IL-6 for implementation of RT-PCR. GAPDH: phosphoglyceraldehyde dehydrogenase and RT-PCR: real time-polymerase chain reaction

\begin{tabular}{lll}
\hline Gene & & Primer sequence \\
\hline GAPDH & Sense & 5'-TGGTATCGTGGAAGGACTCAT-3' \\
& Antisense & 5'-GTGGGTGTCGCTGTTGAAGTC-3' \\
IL-6 & Sense & 5'-GCCACTCACCTCTTCAGAACGA-3' \\
& Antisense & 5'- CTGGCTTGTTCCTCACTACTCT-3' \\
\hline
\end{tabular}


HepG2 cells $\left(1 \times 10^{7}\right.$ cells in $300 \mu \mathrm{l}$ PBS $)$ were subcutaneously injected into the dorsal left flank of 4-week-old male Balb/c nude mice (control and IL-17 KO groups; 5 mice in each group). When the tumor reached approximately $100 \mathrm{~mm}^{2}$, the tumor volume was measured every five days with a microcaliper. Tumor volumes $\left(\mathrm{mm}^{3}\right)$ were estimated by measuring the longest and shortest diameters of the tumors and calculated as previously described. Twenty-five days later, the mice were sacrificed and the tumor weights in the liver were determined.

\section{Orthotopic xenograft liver tumor models}

HepG2 cell lines were stably transfected using the pGL3-control vector (Promega, Madison, WI, USA) and pSV2Neo (ATCC, USA), as previously described [26]. The cells were treated with $10 \mu \mathrm{g}$ of pGL3-control and $1 \mu \mathrm{g}$ of pSV2Neo vectors using Lipofectamine 2000 (Invitrogen) in Opti-MEM (Invitrogen) and then selected with geneticin $(400 \mu \mathrm{g} / \mathrm{mL})$. Stable HepG2 cells expressing Luc were isolated and the clone with the highest Luc expression level (measured by its bioluminescence) was sorted with luciferin (Xenogen, CA, USA) and an in vivo imaging system (Xenogen).

HepG2-Luc cells were dispersed in suspension using pancreatin and the suspension was centrifuged (1000 rpm, $5 \mathrm{~min}$ ) and mixed with PBS before it was pumped into 1-mL sterile syringes. A skin incision was made under the breastbone along the left costal margin. From the incision, muscles and peritoneal layers were bluntly separated to expose part of the left liver. Then, we injected $50 \mu \mathrm{L}$ of cell suspension $\left(1 \times 10^{7}\right.$ tumor cells in $0.3 \mathrm{~mL}$ of PBS) into the left lobe of the liver (both control group and IL-17 KO groups; 3 mice in each group). After regaining consciousness, mice were fed sterilely.

After aqueous solution of luciferin ( $150 \mathrm{mg} / \mathrm{kg}$ intraperitoneally) was injected $10 \mathrm{~min}$ prior to imaging, mice were anesthetized and placed into a light-tight chamber of the CCD camera system (Xenogen). The photons emitted from the luciferase-expressing cells within the animal were quantified for 5 min using Living Image software (Xenogen) as an overlay.

After 10 days of tumor growth, mice were euthanized and their liver tissues were fixed with $10 \%$ formalin. The formalin-fixed tissues were embedded in paraffin, cut into 5 - $\mu \mathrm{m}$ sections, placed onto glass slides, and used to count the total number of tumors with hematoxylin and eosin (H\&E, Sigma-Aldrich, St. Louis, MO, USA) for histological examination. The concentrations of IL-6, MCP-1, CCL5 and VEGF in the liver were detected with an ELISA kit according to the manufacturer's instructions.

\section{Statistical Analysis}

SPSS19.0 was used to analyze the results, which are expressed as the median (percent or mean \pm standard deviation (SD). When two groups were compared, the unpaired Student's t-test was used. When multiple groups were compared, multivariate analysis of variance (ANOVA) was used. A Mann-Whitney U test was used to calculate statistical significance. $P<0.05$ was considered indicative of a significant difference.

\section{Results}

IL-17 enhances the proliferation and migration of HepG2 cells

The CCK-8 assay was used to detect the proliferation of HepG2 cells after treatment with different concentrations of IL-17. As shown in Fig. 1A, IL-17 accelerated the cell proliferation in a concentration-dependent manner with a remarkable growth rate in the IL-17 concentration of $100 \mathrm{ng} / \mathrm{mL}$. Hence, we selected $100 \mathrm{ng} / \mathrm{mL}$ IL-17 in the subsequent experiments. The Transwell assay was used to measure the migration of HepG2 cells. As shown in Fig. 1B, exogenous IL-17 (100 ng/mL treatment for $24 \mathrm{~h})$ remarkably enhanced the migration of HepG2 cells compared to the Control group $(P<0.05)$. The results indicated that IL-17 can promote the proliferation and migration of HepG2 cells.

\section{IL-17 promotes STAT3 phosphorylation}

It is well known that the STAT3 pathway plays a significant role in maintaining hepatocellular carcinoma [27]. Therefore, we predicted that IL-17 might maintain the selfrenewal of HepG2 cells through the STAT3 pathway. To verify this hypothesis, we conducted Western Blot and immunofluorescence analyses. As shown in Fig. 2A, IL-17 enhanced the 
level of phosphorylation of STAT3, while the total STAT3 level was unchanged. Next, we used STAT3 inhibitor, S31-201, or combined utilization of IL-17 (100 ng/mL) and S31-201 to treat HepG2 cells and found that S31-201 could efficiently block the phosphorylation of STAT3, even in the presence of IL-17. Therefore, exogenous IL-17 could not reverse the inhibition effects of S31-201. Immunofluorescence results confirmed the Western Blot results (Fig. 2B). The cytokines associated with the STAT3 pathway were measured using an ELISA. The ELISA results shown in Fig. 2C revealed IL-17 treatment increased the expression levels of MCP-1,

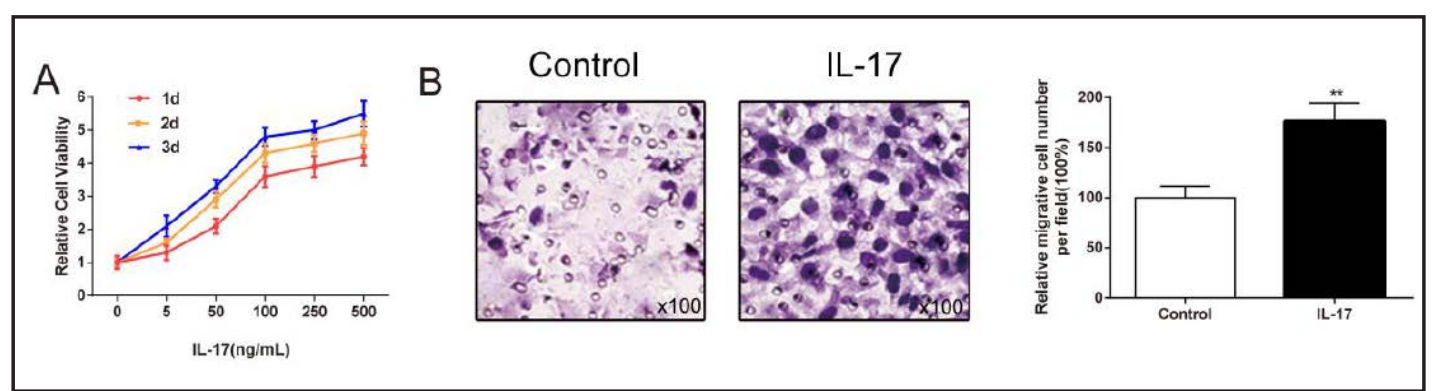

Fig. 1. IL-17 enhances the proliferation and migration of HepG2 cells. (A) Effects of IL-17 on the proliferation of HepG2 cells were detected using the CCK-8 assay. (B) Effects of IL-17 on the migration of HepG2 cells were detected by a Transwell assay (magnification, $\times 100$ ). Data are presented as the mean \pm SD for three independent experiments. ${ }^{* *} P<0.05$ compared to Control.

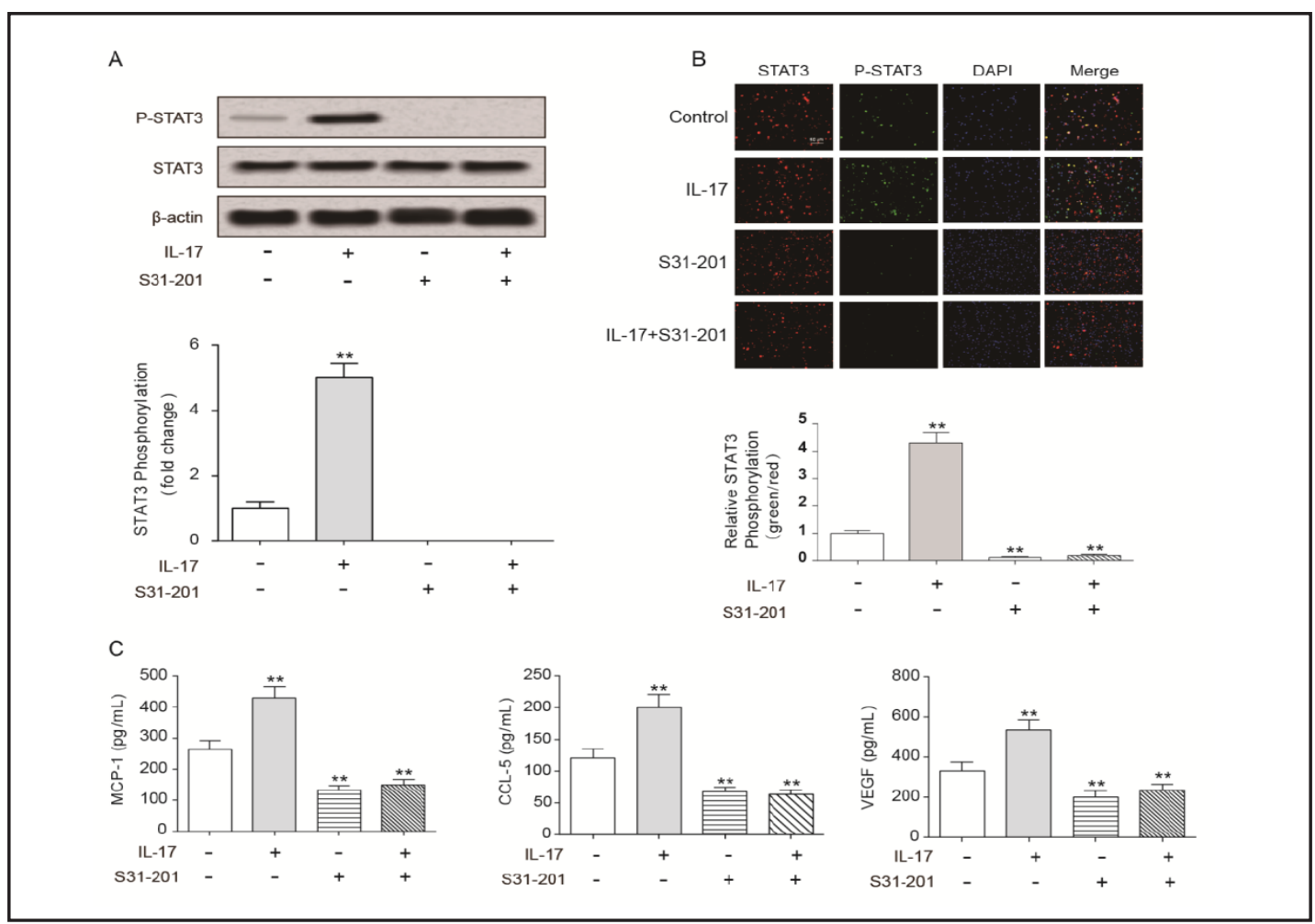

Fig. 2. IL-17 activates the phosphorylation of STAT3. (A) Western Blot analysis of p-STAT3 and STAT3 expression in HepG2 cells after treatment with IL-17 and S31-201. (B) Quantitation of p-STAT3 and STAT3 protein expression using immunofluoresence microscopy in HepG2 cells with IL-17 and S31-201 treatment; p-STAT3 was stained green, STAT3 was stained red and cell nuclei were stained with DAPI. (C) ELISA performed to detect the level of cytokines related to the STAT3 pathway, including MCP-1, CCL5 and VEGF. Data are presented as the mean \pm SD for three independent experiments. ${ }^{* *} P<0.05$ compared to Control. Scale bar $=50 \mu \mathrm{m}$. 


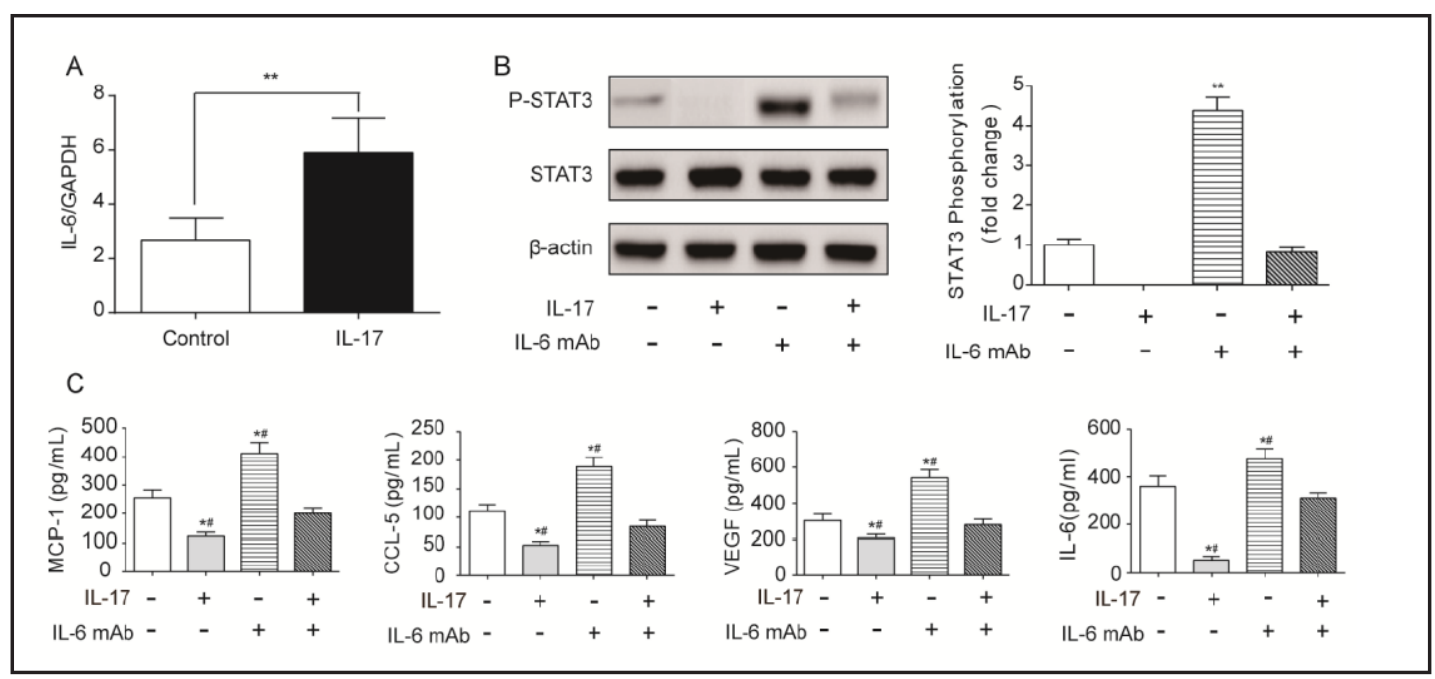

Fig. 3. IL-17 activates the STAT3 pathway through IL-6 (A) The effects of IL-17 on the mRNA expression of IL-6 in HepG2 cells were detected by the RT-qPCR assay. (B) Western Blot analysis of p-STAT3 and STAT3 expression in HepG2 cells after treatment with IL-17 and IL-6 mAb. (C) ELISA performed to detect the levels of IL-6, MCP-1, CCL5 and VEGF. Data are presented as the mean \pm SD for three independent experiments. ${ }^{* *}$ $P<0.05$ compared to Control.

CCL-5 and VEGF compared with the control group $(P<0.05)$, while the inhibitor of STAT3 reduced the levels of MCP-1, CCL-5 and VEGF $(P<0.05)$. Treatment of IL-17 could efficiently attenuate the inhibition of S31-201 on the indicated cytokines, which further verified the activation of STAT3 in the IL-17 treatment group.

\section{IL-17 regulated the STAT3 pathway through IL-6}

To investigate whether IL-17 regulated the STAT3 pathway through producing IL-6, we detected the expression of IL- 6 in the presence or absence of IL-17. The expression of IL-6 mRNA was measured by RT-qPCR (Fig. 3A), and it was shown that IL-17 promotes the expression of IL-6 $(P<0.05)$.

As before, we showed that IL-17 promotes the expression of p-STAT3. To determine whether the IL-17-mediated STAT3 pathway depended on IL-6, we used IL-6 neutralizing mAb (IL-6 mAb, $20 \mathrm{ng} / \mathrm{mL}$ ), or combined utilization of IL-17 (100 ng/mL) and IL-6 mAb (20 ng / $\mathrm{mL}$ ) to treat HepG2 cells. We found that IL- $6 \mathrm{mAb}$ could efficiently block the phosphorylation of STAT3 in the absence of IL-17. However, exogenous IL-17 could neutralize the inhibition effects of IL-6 mAb (Fig. 3B). The ELISA was also performed and the MCP-1, CCL5, VEGF and IL-6 levels were measured. As shown in Fig. 3C, although IL- 6 mAb significantly suppressed the levels of IL-6, MCP-1, CCL5 and VEGF compared with the control group $(P<0.05)$, IL17 could efficiently relieve the downregulation of IL-6, MCP-1, CCL5 and VEGF, which were tightly related to the STAT3 pathway. Taken together, IL-17 activation of the STAT3 pathway depended on IL-6.

IL-17 promotes the proliferation and migration of HepG2 cells via the IL-6/STAT3 pathway

The CCK- 8 and Transwell assays were used to detect the proliferation and migration of HepG2 cells. As shown in Fig. 4A and 4B, IL-17 significantly accelerated cell proliferation and migration, while S31-201 or IL-6 mAb remarkably attenuated cell proliferation and migration compared to Control cells $(P<0.05)$. Meanwhile, IL-6 mAb+IL-17 co-treatment had little effect on cell proliferation and migration compared to Control cells $(P>0.05)$, but S31-201+IL-17 co-treatment significantly attenuated cell proliferation and migration $(P<$ $0.05)$. The results indicated that IL-17 can promote the proliferation and migration of HepG2 cells, but STAT3 inhibitor S31-201 or IL-6 mAb significantly reversed the promoting effects 


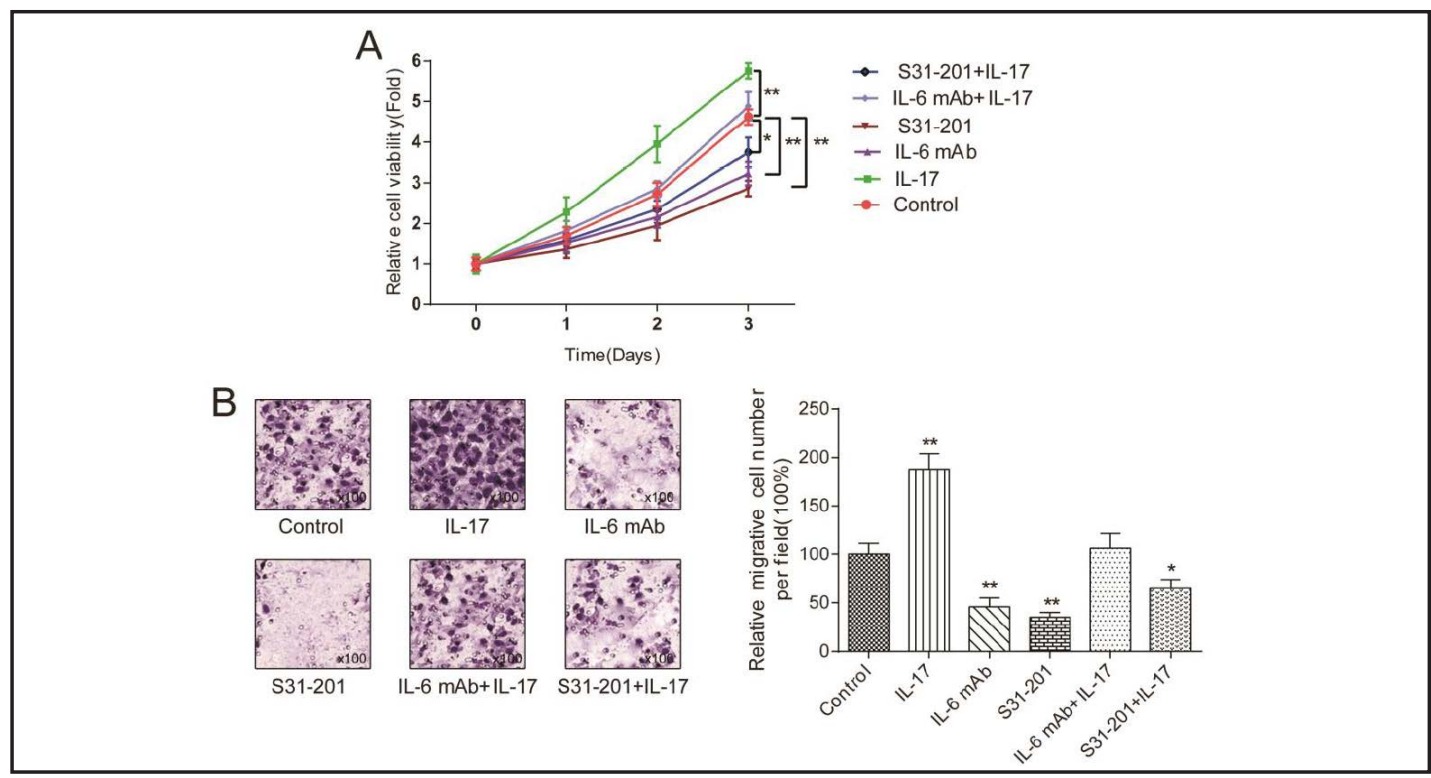

Fig. 4. IL-17 enhances the proliferation and migration of HepG2 cells via the IL-6/STAT3 pathway. (A) Effects of the IL-6/STAT3 pathway on the proliferation of HepG2 cells were detected using CCK-8 assay. (B) Effects of the IL-6/STAT3 pathway on the migration of HepG2 cells were detected by a Transwell assay (magnification, $\times 100$ ). Data are presented as the mean \pm SD for three independent experiments. ${ }^{*} P<0.05$ and ${ }^{* *} P<0.01$ compared to Control.

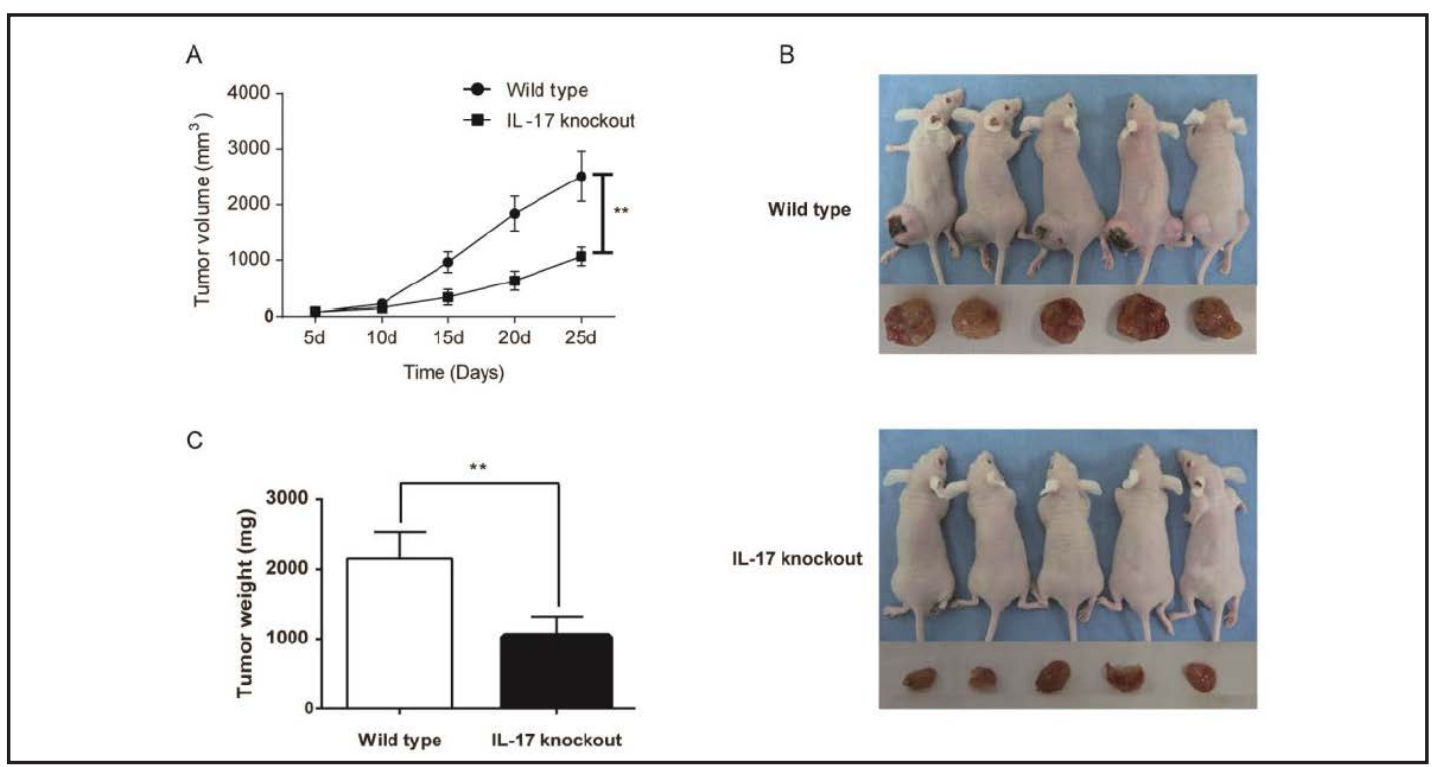

Fig. 5. IL-17 knockout suppressed the proliferation of tumor in heterotopic tumor models. (A) Tumor volumes were measured after stable formation. IL-17 knockout suppressed tumor growth in vivo. B: Samples of the xenograft models at 25 days. C: Mice were sacrificed 25 days later, and tumors were extracted and weighed. IL-knockout decreased the tumor weight in vivo. Data are presented as the mean \pm SD. ${ }^{* *} P<0.05$ compared to wild type mice.

of proliferation and migration from IL-17 stimulation in HepG2 cells $(P<0.05)$. According to those results, we verified that IL-17 activation of the STAT3 pathway depended on IL-6; therefore, IL-6/STAT3 activation was suggested to be responsible for the promoting effects of IL-17 in the proliferation and migration of HepG2 cells. 
Effects of the IL-17/IL-6/STAT3 signaling pathway on the tumor proliferation in mice

To investigate whether IL-17 enhances HepG2 cell proliferation in vivo, we injected HepG2 cells or luciferase-labeled HepG2 cells into nude mice (wild type and IL-17 knockout mice). HepG2 cells were subcutaneously injected into the dorsal left flank of Balb/c mice to construct heterotopic xenograft models. After the tumor was stably $100 \mathrm{~mm}^{3}$ in size, the tumor growth was measured with a microcaliper, as shown in Fig. 5A. The tumor volume in wild type mice increased more quickly than in IL-17 knockout mice $(P<0.05)$. Mice were sacrificed at 25 days. The tumor samples are shown in Fig. 5B, and the tumor weights are compared in Fig. 5C. The tumor volume and weight were higher in wild type mice than IL17 knockout mice $(P<0.05)$. That is, IL-17 gene knockout attenuated tumor growth in vivo.

HepG2-Luc cells were injected into the left lobe of the liver to construct orthotopic xenograft models. Living images of mice at 10 day post injection are shown in Fig. 6A. The sum of the tumor tissues from liver sections in wild type and IL-17 knockout mice were calculated. As shown in Fig. 6B, IL-17 significantly attenuated tumorigenesis in vivo. Liver histological examination was performed, and the density and size of liver tumor tissues of wild mice were higher than for IL-17 knockout mice (Fig. 6C). Total protein was extracted from mice livers; then, the expression of STAT3 and p-STAT3 were quantitatively tested by Western Blot. As shown in Fig. 6D, decreased phosphorylation of STAT3 was evident, while the total STAT3 was unchanged. The concentrations of IL-6, MCP-1, CCL5 and VEGF were detected by ELISA. The results shown in Fig. 6E revealed that the concentrations of IL-6, MCP-1, CCL5 and VEGF were significantly downregulated in IL-17 knockout mice compared with the concentrations in wild type mice $(P<0.05)$. The lower expression of these cytokines in the IL-17 knockout group also verified the decreasing activation of the STAT3 pathway by IL-17 knockout in vivo.

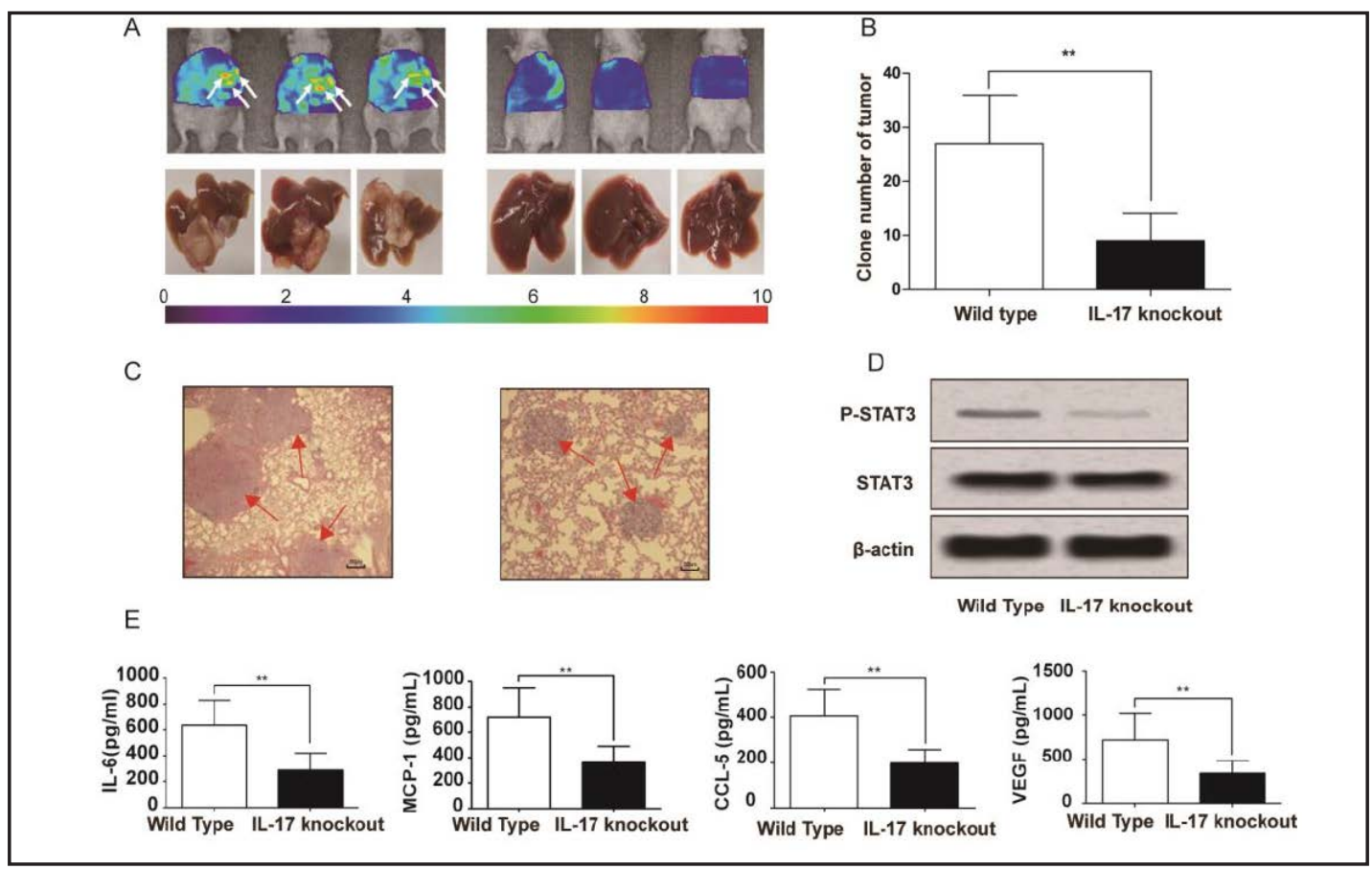

Fig. 6. IL-17 knockout suppressed tumor proliferation in orthotopic tumor models. (A) Samples of the xenograft models at 10 days after injection. (B) The tumor numbers were calculated, and IL-17 attenuated tumorigenesis in vivo. (C) Effects of IL-17 knockout on the density of liver tumor were detected by liver histological examination. (D) Effects of IL-17 knockout on the expression of p-STAT3 in vivo were detected by Western Blot. (E) Effects of IL-17 knockout on the expression levels of IL-6, MCP-1, CLL5 and VEGF in vivo were detected by ELISA. Data are presented as the mean $\pm \mathrm{SD} .{ }^{* *} P<0.05$ compared to wild type mice.

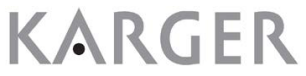




\section{Cellular Physiology Cell Physiol Biochem 2017;43:2379-2390 \\ \begin{tabular}{ll|l} 
and Biochemistry Published online: October 27, 2017 & $\begin{array}{l}\text { (c) } 2017 \text { The Author(s). Published by S. Karger AG, Basel } \\
\text { www.karger.com/cpb }\end{array}$ \\
\hline
\end{tabular} \\ Hu et al.: IL-17/IL-6/STAT3 in HBV-Related HCC}

\section{Discussion}

Hepatocellular carcinoma (HCC), the fifth most common tumor, is the third most common cause of death related to cancer worldwide [28]. Chronic inflammatory states often occur after the development of HCC because of cirrhosis and chronic hepatitis stimulated by either hepatitis B/C virus or non-viral-correlated factors, including alcohol and obesity [29]. Convincing evidence has shown that the immune microenvironment around the liver in HCC could play a significant role in the proliferation, migration, and survival of cancer cells [30].

IL-17 is a primary cell factor secreted by Th17 cells, which plays a crucial role in promoting tumor growth [31]. Although the exact mechanism was not well clarified, many publications reported that IL-17 could exert a notable effect on promoting the growth of cervical tumor, non-small cell carcinoma, breast cancer, colon cancer and papilloma [3235]. Zhang et al. demonstrated that IL-17+ T cells were discovered in large numbers within HCC and were associated with poor survival and improved recurrence, suggesting that Th17 cells and IL-17 could facilitate HCC progression [36]. However, the direct effect and potential mechanism of IL-17 in regulating human HCC cell growth remain incompletely defined. In the present study, we found that IL-17 could facilitate the proliferation and migration of HCC in vitro and in IL-17\% mice, which was similar to the finding by Zhang et al. [36].

STAT3 is a cancer gene that is activated by IL-17 in tumor or stromal cells, which directly enhances tumor growth and metastasis [37]. IL-17 was reported to modulate signaling through distinct pathways, such as the MAPK, NF-kB, and STAT3 pathways, in various inflammatory and tumor cells [38]. In our study, we found that IL-17 could increase the phosphorylation of STAT3 and promote STAT3 activation in vitro and in IL-17\% mice, which was consistent with findings of Wang et al. and Gu et al. [24, 39].

IL-6 is a STAT3 activating factor that is increased in numerous cancers [40]. IL-6 and other members of the IL- 6 family, which can result in cancer-promoting inflammation by inducing the JAK-STAT3 pathway, have been widely reported [41]. Additionally, IL-6 plays an important role in promoting the phosphorylation of both STAT3 and Jak2 [42]. Activation of the STAT3 by phosphorylation in response to IL6 turns on the transcriptions of many downstream genes involved in normal hepatic development [43]. The IL-6-induced STAT3 signaling pathway in tumor cells enhances proliferation, proangiogenic, and antiapoptotic genes. It also leads to inhibition of several pro-inflammatory genes [44]. Therefore, we investigated whether IL-6 caused IL-17-driven IL-6 induction of STAT3 activation in HCC. Then, we found that IL-17 could increase the expression level of IL-6. Furthermore, anti-IL-6 antibodies abrogated IL-17-induced STAT3 activation. These results elucidated that IL-17 could activate STAT3 by promoting the expression of IL- 6 in HCC, which was consistent with the findings of Wang et al. and Gu et al. [24, 39].

Recently, substantial research found that IL-17 could positively induce elevation of IL-6 expression and activation of STAT3 in fibroblasts and inflammatory cells surrounding autoimmune diseases [45]. Wang et al. suggested that IL-17 exerted a direct effect on facilitating the growth of tumor cells via activating the IL-6/STAT3 pathway by improving the expression of IL-6 and a signal transducer in murine B16 melanoma and MB49 bladder carcinoma [39]. Gu et al. suggested that IL-17 exerted a direct effect on promoting HCC growth through the inducing the IL-6/JAK2/STAT3 by activating the AKT pathway [24]. In agreement with the above findings, we clarified the related molecular mechanism of IL-17 in the proliferation and migration of HCC cells in the present study, suggesting that IL-17 may play a significant role in facilitating tumor growth in HCC by activating the IL-17/IL-6/STAT3 pathway in vitro and in IL-17\% mice.

Excluding the above findings about the effect of IL-17 in facilitating tumor growth, our findings contrasted with those in other reports that IL-17 could have an antitumor effect for various tumors. For example, Kortylewski et al. found that tumor growth of MC38 sarcoma 
was promoted in IL-17\% mice [46]. These findings demonstrated that the action of IL-17 in tumor highly depended on the cell type and environment background in modulating the proliferation and metastasis of tumor cells, supporting that the role of IL-17 in HCC requires specific investigation. Further research is needed to discuss the direct effect and mechanism of IL-17 on the biological behavior of tumor cells as well as to clarify why IL-17 has contrasting roles in regulating tumor growth in distinct tumor systems.

Taken together, we have confirmed that IL-17 could facilitate tumor growth. Moreover, our study demonstrated that the tumor-promoting effect mediated by IL-17 might be correlated with a direct action on tumor cells through inducing IL-6, which could activate STAT3 in HCC. Our results indicated that IL-17, STAT3 and IL-6 might act as molecular targets for the diagnosis, treatment and prognosis of hepatitis B virus-related HCC.

\section{Funding}

This study is supported by the following projects: (1) The Joint Special Project Sponsored by Science and Technology Department of Yunnan Province and Kunming Medical University, (2) The Postdoctoral Fellow Special Project of The First People's Hospital of Kunming City, (3) The Foundation for Outstanding Young Scientist in Kunming City, (4) The Ten-HundredThousand Project and The Foundation for Clinical Experts Sponsored by Kunming Municipal Health and Planning Commission, and (5) Yunnan Province Applied Basic Research [N0. 2017FE468 (-099)].

\section{Disclosure Statement}

None.

\section{References}

1 Jemal A, Bray F, Center MM, Ferlay J, Ward E, Forman D: Global cancer statistics. CA Cancer J Clin 2011;61:69-90.

2 El-Serag HB: Hepatocellular carcinoma. N Engl J Med 2011;365:1118-1127.

3 Chuang SC, La Vecchia C, Boffetta P: Liver cancer: descriptive epidemiology and risk factors other than HBV and HCV infection. Cancer Lett 2009;286:9-14.

4 Chang MH: Prevention of hepatitis B virus infection and liver cancer. Recent Results Cancer Res 2014;193:75-95.

-5 Wang B, Zhao XP, Fan YC, Zhang JJ, Zhao J, Wang K: IL-17A but not IL-22 suppresses the replication of hepatitis B virus mediated by over-expression of MxA and OAS mRNA in the HepG2.2.15 cell line. Antiviral Res 2013;97:285-292.

-6 Zhang G, Li Z, Han Q, Li N, Zhu Q Li F, Lv Y, Chen J, Lou S, Liu Z: Altered TNF-alpha and IFN-gamma levels associated with PD1 but not TNFA polymorphisms in patients with chronic HBV infection. Infect Genet Evol 2011;11:1624-1630.

7 He G, Yu GY, Temkin V, Ogata H, Kuntzen C, Sakurai T, Sieghart W, Peck-Radosavljevic M, Leffert HL, Karin M: Hepatocyte IKKbeta/NF-kappaB inhibits tumor promotion and progression by preventing oxidative stressdriven STAT3 activation. Cancer Cell 2010;17:286-297.

-8 Lee JH, Kim C, Baek SH, Ko JH, Lee SG, Yang WM, Um JY, Sethi G, Ahn KS: Capsazepine inhibits JAK/STAT3 signaling, tumor growth, and cell survival in prostate cancer. Oncotarget 2016.

-9 Lee JH, Kim JE, Kim BG, Han HH, Kang S, Cho NH: STAT3-induced WDR1 overexpression promotes breast cancer cell migration. Cell Signal 2016;28:1753-1760.

10 Subramaniam A, Shanmugam MK, Ong TH, Li F, Perumal E, Chen L, Vali S, Abbasi T, Kapoor S, Ahn KS, Kumar AP, Hui KM, Sethi G: Emodin inhibits growth and induces apoptosis in an orthotopic hepatocellular carcinoma model by blocking activation of STAT3. Br J Pharmacol 2013;170:807-821. 


\section{Cellular Physiology Cell Physiol Biochem 2017;43:2379-2390

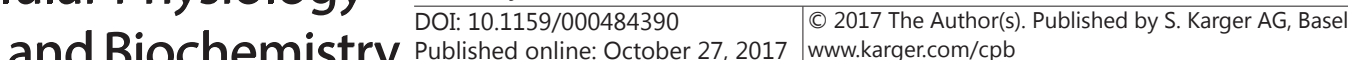 and Biochemistry Published online: October 27, 2017 www.karger.com/cpb}

Hu et al.: IL-17/IL-6/STAT3 in HBV-Related HCC

11 Paul A, Das S, Das J, Samadder A, Bishayee K, Sadhukhan R, Khuda-Bukhsh AR: Diarylheptanoidmyricanone isolated from ethanolic extract of Myrica cerifera shows anticancer effects on HeLa and PC3 cell lines: signalling pathway and drug-DNA interaction. J Integr Med 2013;11:405-415.

12 He G, Karin M: NF-kappaB and STAT3 - key players in liver inflammation and cancer. Cell Res 2011;21:159168.

13 Liu Z, Chen T, Lu X, Xie H, Zhou L, Zheng S: Overexpression of variant PNPLA3 gene at I148M position causes malignant transformation of hepatocytes via IL-6-JAK2/STAT3 pathway in low dose free fatty acid exposure: a laboratory investigation in vitro and in vivo. Am J Transl Res 2016;8:1319-1338.

-14 Park EJ, Lee JH, Yu GY, He G, Ali SR, Holzer RG, Osterreicher CH, Takahashi H, Karin M: Dietary and genetic obesity promote liver inflammation and tumorigenesis by enhancing IL-6 and TNF expression. Cell 2010;140:197-208.

15 Miller AM, Wang H, Bertola A, Park O, Horiguchi N, Ki SH, Yin S, Lafdil F, Gao B: Inflammation-associated interleukin-6/signal transducer and activator of transcription 3 activation ameliorates alcoholic and nonalcoholic fatty liver diseases in interleukin-10-deficient mice. Hepatology 2011;54:846-856.

-16 Arababadi MK, Bidaki MZ, Kennedy D: IL-17A in hepatitis B infection: friend or foe? Arch Virol 2014;159:1883-1888.

17 Liu QL, Huang L, Zhao QJ, Li Q, He Z: Relationship between serum interleukin-17 level and inflammatory bowel disease. J Biol Regul Homeost Agents 2016;30:181-188.

18 Schminke B, Trautmann S, Mai B, Miosge N, Blaschke S: Interleukin 17 inhibits progenitor cells in rheumatoid arthritis cartilage. Eur J Immunol 2016;46:440-445.

19 Axtell RC, de Jong BA, Boniface K, van der Voort LF, Bhat R, De Sarno P, Naves R, Han M, Zhong F, Castellanos JG, Mair R, Christakos A, Kolkowitz I, Katz L, Killestein J, Polman CH, de Waal Malefyt R, Steinman L, Raman C: T helper type 1 and 17 cells determine efficacy of interferon-beta in multiple sclerosis and experimental encephalomyelitis. Nat Med 2010;16:406-412.

-20 Yang LJ, Gao W, Bai JY, Zhang XK, Han X, Sun YH, Zhang LL, Zhang MM: Correlation between Interleukin-17 gene polymorphism and gastric cancer susceptibility in Han Chinese population. Eur Rev Med Pharmacol Sci 2016;20:1271-1282.

21 Xiang T, Long H, He L, Han X, Lin K, Liang Z, Zhuo W, Xie R, Zhu B: Interleukin-17 produced by tumor microenvironment promotes self-renewal of CD133+ cancer stem-like cells in ovarian cancer. Oncogene 2015;34:165-176.

22 Su X, Ye J, Hsueh EC, Zhang Y, Hoft DF, Peng G: Tumor microenvironments direct the recruitment and expansion of human Th17 cells. J Immunol 2010;184:1630-1641.

23 Li J, Lau GK, Chen L, Dong SS, Lan HY, Huang XR, Li Y, Luk JM, Yuan YF, Guan XY: Interleukin 17A promotes hepatocellular carcinoma metastasis via NF-kB induced matrix metalloproteinases 2 and 9 expression. PLoS One 2011;6:e21816.

24 Gu FM, Li QL, Gao Q, Jiang JH, Zhu K, Huang XY, Pan JF, Yan J, Hu JH, Wang Z, Dai Z, Fan J, Zhou J: IL-17 induces AKT-dependent IL-6/JAK2/STAT3 activation and tumor progression in hepatocellular carcinoma. Mol Cancer 2011;10:150.

-25 Gu Y, Lin S, Li JL, Nakagawa H, Chen Z, Jin B, Tian L, Ucar DA, Shen H, Lu J, Hochwald SN, Kaye FJ, Wu L: Altered LKB1/CREB-regulated transcription co-activator (CRTC) signaling axis promotes esophageal cancer cell migration and invasion. Oncogene 2012;31:469-479.

-26 Edinger M, Sweeney TJ, Tucker AA, Olomu AB, Negrin RS, Contag CH: Noninvasive assessment of tumor cell proliferation in animal models. Neoplasia 1999;1:303-310.

-27 Zhao H, Guo Y, Li S, Han R, Ying J, Zhu H, Wang Y, Yin L, Han Y, Sun L, Wang Z, Lin Q Bi X, Jiao Y, Jia H, Zhao J, Huang Z, Li Z, Zhou J, Song W, Meng K, Cai J: A novel anti-cancer agent Icaritin suppresses hepatocellular carcinoma initiation and malignant growth through the IL-6/Jak2/Stat3 pathway. Oncotarget 2015;6:31927-31943.

28 El-Serag HB, Rudolph KL: Hepatocellular carcinoma: epidemiology and molecular carcinogenesis. Gastroenterology 2007;132:2557-2576.

29 Hagemann T, Balkwill F, Lawrence T: Inflammation and cancer: a double-edged sword. Cancer Cell 2007;12:300-301.

- 30 Ferrone C, Dranoff G: Dual roles for immunity in gastrointestinal cancers. J Clin Oncol 2010;28:4045-4051.

-31 Miossec P, Korn T, Kuchroo VK: Interleukin-17 and type 17 helper T cells. N Engl J Med 2009;361:888-898. 


\section{Cellular Physiology Cell Physiol Biochem 2017;43:2379-2390

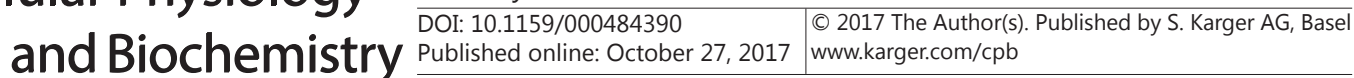

Hu et al.: IL-17/IL-6/STAT3 in HBV-Related HCC

-32 Tartour E, Fossiez F, Joyeux I, Galinha A, Gey A, Claret E, Sastre-Garau X, Couturier J, Mosseri V, Vives V, Banchereau J, Fridman WH, Wijdenes J, Lebecque S, Sautes-Fridman C: Interleukin 17, a T-cell-derived cytokine, promotes tumorigenicity of human cervical tumors in nude mice. Cancer Res 1999;59:36983704.

-33 Numasaki M, Watanabe M, Suzuki T, Takahashi H, Nakamura A, McAllister F, Hishinuma T, Goto J, Lotze MT, Kolls JK, Sasaki H: IL-17 enhances the net angiogenic activity and in vivo growth of human non-small cell lung cancer in SCID mice through promoting CXCR-2-dependent angiogenesis. J Immunol 2005;175:61776189.

-34 Nam JS, Terabe M, Kang MJ, Chae H, Voong N, Yang YA, Laurence A, Michalowska A, Mamura M, Lonning S, Berzofsky JA, Wakefield LM: Transforming growth factor beta subverts the immune system into directly promoting tumor growth through interleukin-17. Cancer Res 2008;68:3915-3923.

-35 Xiao M, Wang C, Zhang J, Li Z, Zhao X, Qin Z: IFNgamma promotes papilloma development by up-regulating Th17-associated inflammation. Cancer Res 2009;69:2010-2017.

-36 Zhang JP, Yan J, Xu J, Pang XH, Chen MS, Li L, Wu C, Li SP, Zheng L: Increased intratumoral IL-17-producing cells correlate with poor survival in hepatocellular carcinoma patients. J Hepatol 2009;50:980-989.

37 Gravie JF: [Treatment of recurrent rectal prolapse]. Gastroenterol Clin Biol 2008;32:S235-239. Gaffen SL: Structure and signalling in the IL-17 receptor family. Nat Rev Immunol 2009;9:556-567. Wang L, Yi T, Kortylewski M, Pardoll DM, Zeng D, Yu H: IL-17 can promote tumor growth through an IL-6Stat3 signaling pathway. J Exp Med 2009;206:1457-1464.

40 Hirano T, Ishihara K, Hibi M: Roles of STAT3 in mediating the cell growth, differentiation and survival signals relayed through the IL-6 family of cytokine receptors. Oncogene 2000;19:2548-2556.

41 Yu H, Pardoll D, Jove R: STATs in cancer inflammation and immunity: a leading role for STAT3. Nat Rev Cancer 2009;9:798-809.

-42 Aggarwal S, Lam A, Bolisetty S, Carlisle MA, Traylor A, Agarwal A, Matalon S: Heme Attenuation Ameliorates Irritant Gas Inhalation-Induced Acute Lung Injury. Antioxid Redox Signal 2016;24:99-112.

43 Jung IH, Choi JH, Chung YY, Lim GL, Park YN, Park SW: Predominant Activation of JAK/STAT3 Pathway by Interleukin-6 Is Implicated in Hepatocarcinogenesis. Neoplasia 2015;17:586-597.

44 Yu H, Kortylewski M, Pardoll D: Crosstalk between cancer and immune cells: role of STAT3 in the tumour microenvironment. Nat Rev Immunol 2007;7:41-51.

-45 Ogura H, Murakami M, Okuyama Y, Tsuruoka M, Kitabayashi C, Kanamoto M, Nishihara M, Iwakura Y, Hirano T: Interleukin-17 promotes autoimmunity by triggering a positive-feedback loop via interleukin-6 induction. Immunity 2008;29:628-636.

-46 Kortylewski M, Xin H, Kujawski M, Lee H, Liu Y, Harris T, Drake C, Pardoll D, Yu H: Regulation of the IL-23 and IL-12 balance by Stat3 signaling in the tumor microenvironment. Cancer Cell 2009;15:114-123. 